\title{
Zinc Sulphate in the Treatment of Cutaneous Leishmaniasis: an in Vitro and Animal Study
}

\author{
Rafid A Najim/+ , Khalifa E Sharquie*, Imad B Farjou
}

\author{
Department of Pharmacology * Department of Dermatology, College of Medicine, University of Baghdad, \\ P.O. Box 61208, Postal code 12114, Baghdad, Iraq
}

This study was designed to evaluate the effectiveness of zinc sulphate both in vitro and in an animal model against both strains of old world cutaneous leishmaniasis. The in vitro sensitivities of promastigotes and axenic amastigotes of both Leishmania major and $\mathrm{L}$. tropica to zinc sulphate was determined, the $L D_{50}$ calculated and compared to the standard treatment for cutaneous leishmaniasis pentavalent antimony compounds. The results show that the two forms of both strains were sensitive to zinc sulphate and their respective $L D_{50}$ were lower compared to the pentavalent antimony compound. Furthermore the sensitivities of the forms of both strains were tested using a simple slide method and compared to results of the standard method. To confirm this result, zinc sulphate was administered orally to mice infected with cutaneous leishmaniasis both therapeutically and prophylactically. Results showed that oral zinc sulphate was effective in both treatment and prophylaxis for cutaneous leishmaniasis. These results encourage the use of oral zinc sulphate in the treatment of cutaneous leishmaniasis clinically.

Key words: cutaneous leishmaniasis - Leishmania major - Leishmania tropica - axenic amastigotes - mice zinc sulphate

There are two modalities for treatment of cutaneous leishmaniasis (CL) namely local and systemic treatment (WHO 1984). There are many effective local modalities for therapy of cutaneous leishmaniasis including physical methods such as using infra red (Rahim \& Tatar 1966, Al-Rubaie 1990), by freezing (Bassiuny et al. 1982) or electrotherapy (Sharquie et al. 1998). Intralesional administration of drugs represent another alternative. The drugs which might be used include pentavalent antimony compounds (WHO 1984, Sharquie et al. 1988, Faris et al. 1993, Sharquie et al. 1997) and hypertonic sodium chloride (Sharquie 1995, Sharquie et al. 1997). Topically applied paromoycin represent yet another novel alternative (ElOn et al. 1988). These methods of treatment are used when there are few lesions (WHO 1984, Sharquie et al. 1988, Sharquie 1995).

On the other hand, systemic therapy for cutaneous leishmaniasis is indicated when there are numerous lesions and/or when lesions cannot be injected intralesionally (WHO 1984, Sharquie 1995). There is no effective systemic treatment for

This paper is based on a PhD Thesis by the first author. ${ }^{+}$Corresponding author.

Received 9 February 1998

Accepted 22 June 1998 cutaneous leishmaniasis apart from pentavalent antimony compounds (WHO 1984, Bryceson 1987). However, these drugs are expensive and may be associated with numerous serious side effects (Desjeux 1992, Neouimine 1996). Ketoconazole, a broad spectrum antifungal, has been reported to be effective in CL (Kurban et al. 1986). However the prolonged period of treatment together with the potential hepatotoxicity may limit its use (Bryceson 1987).

No oral treatment for cutaneous leishmaniasis with proven efficacy exists despite the large number of drugs tested (Bryceson 1987). Most recently zinc sulphate was tested as an intralesional therapy for cutaneous leishmaniasis (Sharquie \& AlAzzawi 1996, Sharquie et al. 1997) and shown to be a very effective treatment with a high cure rate. An in vitro test has indicated the possible sensitivity of Leishmania to zinc sulfate (Sharquie \& AlAzzawi 1996).

Since zinc sulphate administered orally was used for a long time in medicine and dermatology (Neldner 1982a, b) then its use as an oral therapy for cutaneous leishmaniasis might represent an important addition to the armamentarium of antileishmanial drugs. The aim of the present study was to evaluate the anti-leishmanial effect of zinc sulphate both in vitro and in an animal model with the view of using it as an oral treatment for cutaneous leishmaniasis. 


\section{MATERIALS AND METHODS}

Source of parasites - L. major and L. tropica promastigotes were obtained from the Leishmania Unit at The Research Center/Saddam College of Medicine. The parasites, isolated initially from patients with CL, were typed using enzyme electrophoresis. The parasites were serially passaged in Balb/c mice every two months. When supplied they were recently isolated on semi-solid medium. They were maintained on semi-solid medium and subcultured every 21 days.

To infect mice $L$. major promastigotes were used. Promastigotes were isolated primarily on a semi-solid medium and subsequently subcultured. For animal inoculation promastigotes from the third subculture, grown on a liquid medium, were used. Each animal was given $0.5 \times 10^{7}$ promastigotes.

Media - The following media were used: Semisolid medium: it was used for the routine maintenance of the parasite in the laboratory throughout the study. This culture medium was originally described by Adler and Theodor (1926). Biphasic medium: the Nove-MacNeal-Nicolle (NNN) biphasic medium was one of the first media developed for cultivating Leishmania and is still widely used (Jaffe et al. 1987). Liquid medium: the liquid medium used was that described by Al-Bashir et al. (1992) which is a modification of the semi-solid medium of Adler and Theodor (1926).

The parasite forms - Promastigotes: promastigotes of either L. major or L. tropica growing on the semi-solid medium were transferred to biphasic medium. After 7-10 days of incubation at $26^{\circ} \mathrm{C}$, heavy growth was obtained. The liquid phase was transferred to liquid medium and growth was monitored every other day with fresh liquid medium added as required until a sufficient volume of the liquid medium containing the desired number of parasites was obtained. Amastigotes: promastigote to axenic amastigote transformation was carried out according to the procedure described by Al-Bashir et al. (1992) and Al-Ani (1992). Briefly, $25 \mathrm{ml}$ screw-capped vials received $4 \mathrm{ml}$ of liquid medium and $1 \mathrm{ml}$ inoculum from a $\log$ phase promastigote culture maintained on a liquid medium at $26^{\circ} \mathrm{C}$. The initial parasite density was $2 \times 10^{6} \mathrm{p} / \mathrm{ml}$. Cultures were incubated for three days at $35^{\circ} \mathrm{C}$. The culture was then examined and $1 \mathrm{ml}$ of it subcultured in $4 \mathrm{ml}$ of liquid medium as described by Al-Ani (1992). Amastigotes obtained in the third subculture were used to test for drug sensitivity.

Drugs - Zinc sulphate: zinc sulphate heptahydrate $\mathrm{ZnSO}_{4} \cdot 7 \mathrm{H}_{2} \mathrm{O}(\mathrm{BDH})$ was dissolved in distilled water in known concentration and autoclaved at $121^{\circ} \mathrm{C}$ for $20 \mathrm{~min}$ before use. Meglumine antimoniate: ampoules of meglumine antimoniate
(Glucantim®) purchased from Spica, France were used. Sodium stibogluconate: vials of sodium stibogluconate (Pentostam $\left.{ }^{\circledR}\right)$ purchased from Wellcome, England were used.

The effects of drugs on promastigotes and axenic amastigotes of $L$. major and $L$. tropica Promastigotes or axenic amastigotes of either $L$. major or L. tropica growing on liquid medium were used. A fixed initial density of the parasites was transferred to screw-capped vials containing $5 \mathrm{ml}$ of liquid medium to which different concentration of either zinc sulphate or meglumine antimoniate were added. Each run also included a control. Each concentration was done in duplicate. The vials were then incubated at $26^{\circ} \mathrm{C}$ or $35^{\circ} \mathrm{C}$ for promastigotes or axenic amastigotes respectively. On the next four days the culture were counted. A 1:10 dilution in saline together with the appropriate dye was prepared. The dye for promastigotes was $0.4 \%$ Trypan blue. The promastigotes permeable to the blue dye are dead while viable ones exclude the dye (Jaffe et al. 1987). While the dye for axenic amastigotes was $0.4 \%$ Erythrocin $b$, living axenic amastigotes exclude the red dye, dead forms are pink (Jaffe et al. 1987). The chamber of a Neubauer slide is charged and the number of organisms in 16 small corner square are counted. The total number per $\mathrm{ml}=\mathrm{N}$ (counted) $\times 10$ (number in $1 \mathrm{~mm}^{3} \times 10^{3}$ (number $1 \mathrm{ml}$ ) x 10 (dilution factor). The $\mathrm{LD}_{50}$ was calculated according to the method of Hearly (1988).

The slide method - This method was first described by Sharquie (1995). A modified method was used in the present work and results obtained compared with results obtained by the previous method. Promastigotes or axenic amastigotes of $L$. major or $L$. tropica were used. The appropriate dye was added to a dilution of the parasites growing on liquid medium. Trypan blue $0.4 \%$ was used for promastigotes and Erythrocin b for axenic amastigotes. One drop containing the parasites was put on a slide together with a drop of a drug solution and covered by a cover slip. The slides were examined under the microscope and the percentage of stained parasites were noted. Normal saline was used as a control.

Animals - Male Balb/c mice weighing 20-25 g were used. Mice were obtained from the animal house at the Drug Control Center. Animals were housed in groups of five and had free access to laboratory chow and water ad libitum throughout the study. Two animal study groups were prepared: Therapeutic group: forty mice were included in this group. Each mouse had an area $1 \mathrm{~cm}$ above the base of the tail, shaven, sterilized and injected with $5 \times 10^{7}$ L. major promastigotes subcutaneously. Animals were then divided into groups of 10 mice. 
Treatment was commenced immediately after infection. Three groups received either 100, 200 or $400 \mathrm{mg} / \mathrm{kg}$ of zinc sulphate solution orally via a gastric tube. Treatment was continued for five days. Drug concentrations were adjusted so that each mice received $10 \mathrm{ml} / \mathrm{kg}$ body weight. A control group of 10 animals received distilled water (Trotter et al. 1980). Prophylactic group: twenty mice divided into two groups were used. One group of animals received $200 \mathrm{mg} / \mathrm{kg}$ of zinc sulphate orally for five days while a another group was given distilled water for the same period and considered as a control group. On the fifth day mice were infected with $0.5 \times 10^{7} 1 \mathrm{~L}$. major promastigotes on the shaved base of the tail.

On weekly intervals beginning from the second week till the eighth week post-infection mice were assessed for the development of lesion. The scale of Trotter et al. (1980) was used. The $\mathrm{ED}_{50}$ was calculated by the method of Hearly (1988).

\section{RESULTS}

Effect of zinc sulphate against L. major promastigotes - Fig. 1 shows the effect of different concentrations of zinc sulphate on promastigotes of $L$. major. Zinc, in geometrically increasing concentrations, dose dependently inhibited the growth of $L$. major promastigotes. The $\mathrm{LD}_{50}$ for zinc sulphate was $221.9 \mathrm{mg} / \mathrm{ml}$ or $44.4 \mathrm{mg} / \mathrm{ml}$ of $\mathrm{Zn}^{++}$if calculated for zinc (Table). Comparing the effect of zinc to that of the pentavalent antimony compounds the $\mathrm{LD}_{50}$ for antimony is $334.7 \mathrm{mg} / \mathrm{ml} \mathrm{Sb}^{\mathrm{v}}$ (Table).

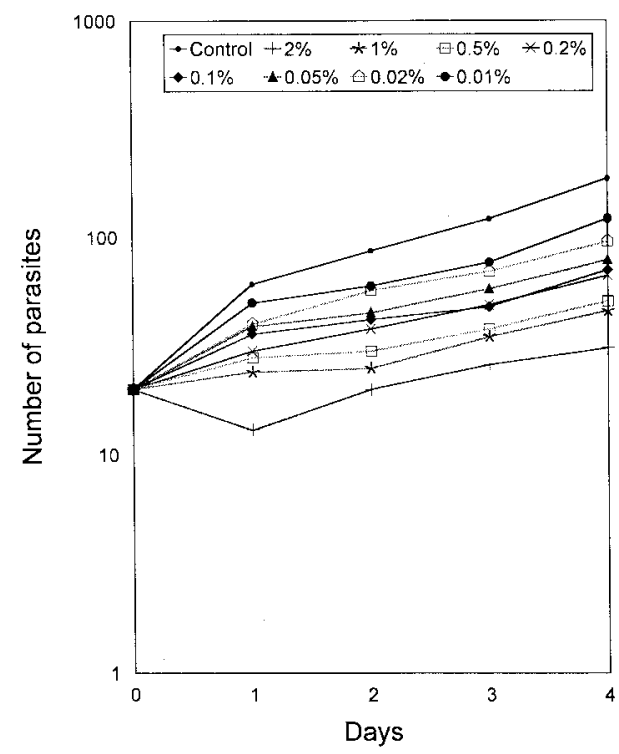

Fig. 1: the effect of different concentrations of zinc sulfate on promastigotes of Leishmania major growing exponentially in liquid media.

\section{TABLE}

The $\mathrm{LD}_{50}$ of zinc and pentavalent antimony against promastigotes and axenic amasgastigotes of Leishmania major and L. tropica

\begin{tabular}{lcc}
\hline Organism & $\mathrm{Zn}^{++}(\mathrm{mg} / \mathrm{ml})$ & $\mathrm{Sb}^{\mathrm{v}}(\mathrm{mg} / \mathrm{ml})$ \\
\hline L. major, promastigotes & 44.4 & 334.7 \\
L. major, axenic amastigotes & 25.4 & 139.2 \\
L. tropica, promastigotes & 32.4 & $>2250$ \\
L. tropica, axenic amastigotes & 27.6 & 163.3 \\
\hline
\end{tabular}

Effect of zinc sulphate against L. major axenic amastigotes - Fig. 2 shows the effect of different concentration of zinc sulphate on axenic amastigotes of L. major. Zinc, in geometrically increasing concentration, dose dependently inhibited the growth of $L$. major axenic amastigotes. It is to be noted that the dose of $20 \mathrm{mg} / \mathrm{ml}$, which gives a $2 \%$ concentration used for intralesional injections, caused $82.3 \%$ inhibition of growth of L. major axenic amastigotes. This effect was noted from the first day (Fig. 2).

The $\mathrm{LD}_{50}$ for zinc sulphate against axenic amastigotes was $126.87 \mathrm{mg} / \mathrm{ml}$ or $25.4 \mathrm{mg} / \mathrm{ml}$ of $\mathrm{Zn}^{++}$(Table). Comparing this effect to $\mathrm{LD}_{50}$ for pentavalent antimony compounds the $\mathrm{LD}_{50}$ was $139.2 \mathrm{mg} / \mathrm{ml}$ of $\mathrm{Sb}^{\mathrm{v}}$ (Table).

Effect of zinc sulphate against promastigotes of L. tropica - Fig. 3 represents the effect of different concentration of zinc sulphate against promastigotes of $L$. tropica. Zinc, in geometrically increasing concentration dose dependently inhibited the growth of promastigotes in vitro.

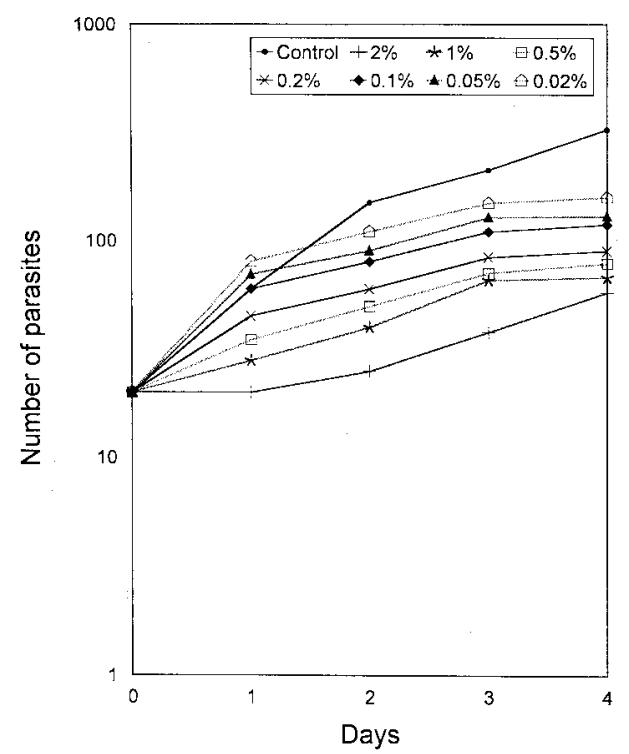

Fig. 2: the effect of different concentrations of zinc sulfate on amastigotes of Leishmania major growing exponentially in liquid media. 


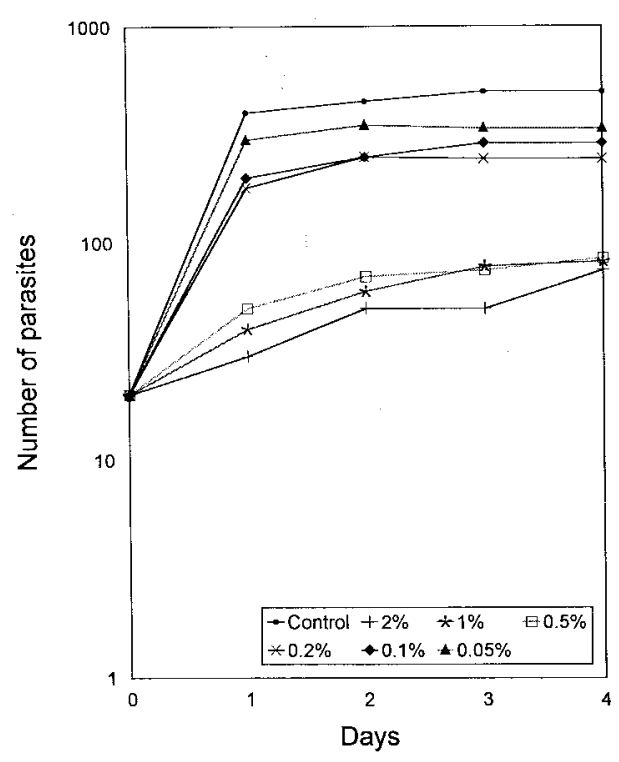

Fig. 3: the effect of different concentrations of zinc sulfate on promastigotes of Leishmania tropica growing exponentially in liquid media.

The $\mathrm{LD}_{50}$ for zinc sulphate was $161.8 \mathrm{mg} / \mathrm{ml}$ or if calculated for zinc is $32.4 \mathrm{mg} / \mathrm{ml}$ of $\mathrm{Zn}^{++}$ (Table). When pentavalent antimony compound was used, then a concentration of $2550 \mathrm{mg} / \mathrm{ml}$ of $\mathrm{Sb}^{\mathrm{v}}$ pentavalent antimony did not produce $50 \%$ inhibition (Table).

Effect of zinc sulphate against axenic amastigotes of L. tropica - Fig. 4 represents the effect of different concentration of zinc sulphate against axenic amastigotes of L. tropica. Zinc in geometrically increasing concentration dose dependently inhibited the growth of $L$. tropica axenic amastigotes in vitro. The $\mathrm{LD}_{50}$ for zinc sulphate was $137.9 \mathrm{mg}$ or $27.8 \mathrm{mg}$ of $\mathrm{Zn}^{++}$(Table). Comparing this to the effect of pentavalent antimony compounds then the $\mathrm{LD}_{50}$ was $163.3 \mathrm{mg}$ of $\mathrm{Sb}^{\mathrm{v}}$.

Slide method - Addition of $2 \%$ zinc sulphate solution to promastigotes of either L. major or $L$. tropica caused immediate paralysis and death of promastigotes as evidenced by their staining blue by Trypan blue. When zinc sulphate was added to axenic amastigotes of both species of Leishmania it caused immediate death as evidenced by the pink stain by Erythrocin $b$ stain. There was no change in the shape of either forms with zinc sulphate.

When sodium stibogluconate or meglumine antimoniate were added to promastigotes of $L$. major or L. tropica then it caused immediate paralysis with shrinkage and this staining blue with Trypan blue stain. Axenic amastigotes both species showed shrinkage and death when sodium stibogluconate or meglumine antimoniate were added. The death was evidenced by the pink stain-

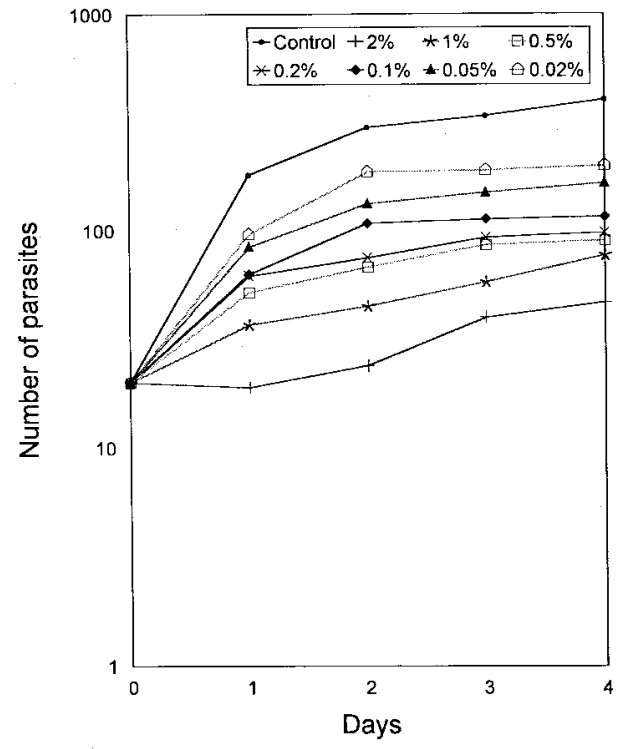

Fig. 4: the effect of different concentrations of zinc sulfate on amastigotes of Leishmania tropica growing exponentially in liquid media.

ing by Erythrocin b stain.

Treatment group - Fig. 5 shows the results of the treatment group. Zinc sulphate administered orally to mice immediately following infection with Leishmania parasites and for five consecutive days resulted in a dose dependent decrease in the mean lesion score of the treated mice when compared to sham treated controls. The $\mathrm{ED}_{50}$ for zinc sulphate from these experiments was $59 \mathrm{mg} / \mathrm{kg}$.

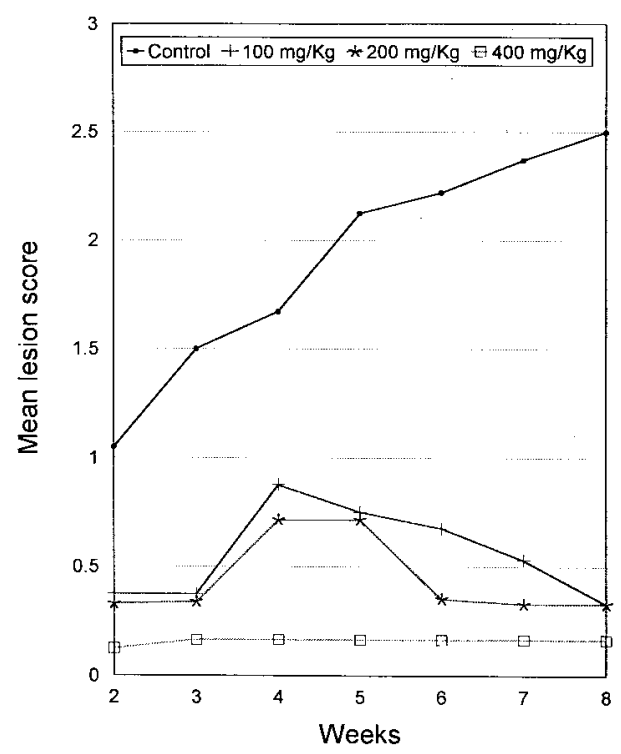

Fig. 5: the mean lesion score in mice infected with cutaneous leishmaniasis and treated with different doses of oral zinc sulfate. 
Prophylactic group - When zinc sulphate was administered for five consecutive days and after the last dose mice were infected with Leishmania parasites then oral zinc sulphate showed a prophylactic role as well (Fig. 6). Thus although there was an increase in the mean lesion score at the first few weeks however the mean lesion score was well below that of the sham treated control at the end of the eight weeks of follow up.

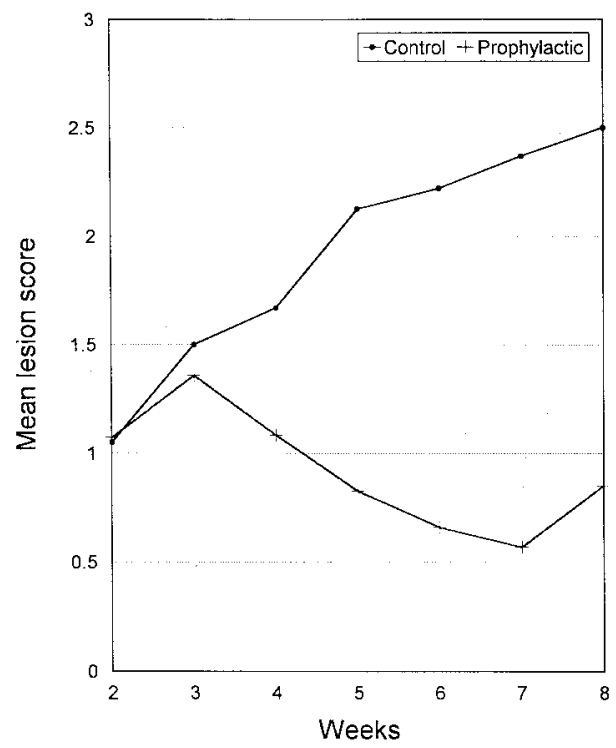

Fig. 6: the mean lesion score in mice which received a prophylactic dose of oral zinc sulfate and were then infected with cutaneous leishmaniasis.

\section{DISCUSSION}

Results of the present investigation clearly indicate that zinc sulphate inhibits the growth of promastigotes of both $L$. major and L. tropica in vitro. To compare the effect of zinc, the effect of the pentavalent antimony compound, meglumine antimoniate was also studied.

There are no reports about the effect of meglumine antimoniate on cutaneous species of Leishmania in vitro. However the other pentavalent antimony compound sodium stibogluconate was reported to have low activity against $L$. major promastigotes in vitro (Neal 1987). This agrees with the results of the present work. No report about the effect of pentavalent antimony compounds on promastigotes of $L$. tropica was found.

It might be argued that promastigotes are of a limited value to study drugs in vitro since parasites infect in the form of intracellular amastigotes. However since this study was the first to investigate the effect of zinc on Leishmania so it was of interest to study the effect of zinc on promastigotes as well as to compare this effect to that of pentavalent antimony compounds. In addition since study of prophylactic role for zinc was planned, the effect of zinc on promastigotes was of interest.

Two models have been utilized in the study of drugs on Leishmania amastigotes till now: the effect of drugs on intracellular amastigotes i.e. inside the macrophages (Neal 1987); the effect of drugs on amastigotes obtained by disrupting macrophages containing the amastigotes which are then grown on a liquid medium. However subsequently amastigotes will grow as promastigotes. Several investigators were able to transform promastigotes into amastigotes and maintain a viable and dividing culture of amastigotes by successive sub culturing of Leishmania promastigotes at $35^{\circ} \mathrm{C}$ (Pan 1984, Pan \& Pan 1986, Al-Bashir et al. 1992). Subsequent investigations have indicated that the amastigotes obtained by the procedure of Al-Bashir et al. (1992) were more similar to intracellular amastigotes than promastigotes. Thus using electron microscopy Al-Ani (1992) has concluded there is no difference between axenic amastigotes and intracellular amastigotes in their fine structure. AlBashir (1992) compared the enzymes of both axenic amastigotes and intracellular amastigotes and concluded that "axenic amastigotes are more physiologically related to in vivo amastigotes than to promastigotes" and suggested that axenic amastigotes may serve as a convenient model system in laboratory investigation including drug effect. Al-Ani (1992) used axenic amastigotes of $L$. donovani to compare the effect of sodium stibogluconate on promastigotes, intracellular amastigotes and axenic amastigotes. He concluded that promastigotes were insensitive to sodium stibogluconate but both axenic and intracellular amastigotes were both sensitive.

In the present investigation it was concluded that the use of intracellular amastigotes i.e. inside macrophages may not be appropriate since zinc has a direct effect on macrophages (Prasad 1979, 1985, Wirth et al. 1989). Therefore in that model one would not be able to differentiate any direct anti leishmanial effect from an indirect effect on macrophages. Since axenic amastigotes continue to grow and divide as amastigotes so it was preferred to the second model. Results obtained with axenic amastigotes clearly indicate that axenic amastigotes are sensitive to the effect of zinc. The fact that the results of this work was used as a base for administration of zinc sulphate to patients with cutaneous leishmaniasis give support to the model of amastigotes for studying drug effects (Sharquie et al. 1997). 
At this stage, the exact mechanism of the anti leishmanial effect of zinc can only be speculated. It is well known that zinc plays an important role in specific metalloenzyme systems concerned with nucleic acid and protein synthesis (Cousin 1985). Furthermore zinc has been reported to inhibit DNA polymerase of herpes simplex virus (Fridlender et al. 1978). Therefore, we may speculate that a very probable site of action of zinc is on some enzymes concerned with nucleic acid metabolism. However further investigation is needed to confirm the exact mechanism of action of zinc against Leishmania.

The slide method was first used by Sharquie (1995) to test the effect of hypertonic sodium chloride on CL. It is a very simple and quick method, but drug concentrations cannot be controlled exactly. However this situation may be analogous to intralesional treatment of CL whereby the drug is given in full concentration directly into the lesion without taking the dose into consideration. In the present investigation, this method was further developed. Thus an indicator dye was added so as to indicate the death of the parasite instead of depending on the motility as an indicator of the viability of parasite. The use of amastigotes as well as promastigotes is another addition in order to make this method more akin to the clinical situation.

The results obtained with $2 \%$ zinc sulphate agrees with the results obtained in clinical trials (Sharquie \& Al-Azzawi 1996, Sharquie et al. 1997). A very high rate of inhibition of Leishmania parasites was obtained by both methods.

However, the results obtained with pentavalent antimony compounds are different from the results obtained by addition of drug to the culture media since in this method complete immobilization was obtained when a drop of pentavalent antimony compound was added. But it must be kept in mind that we are using a hypertonic solution which is higher than any drug concentration used. This high concentration has an osmotic effect (Sharquie 1995). This mechanism of action may be added to the other mechanism of action of pentavalent antimony compounds when they are given intralesionally. This may account for the fact that we need one or two injection intralesionally of the pentavalent antimony compounds to achieve cure while we needed a much longer course if the drug is given systemically.

It might be concluded that this simple method might give quick results about the efficacy of intralesionally administered drugs. It would be interesting to compare the effect of different drugs which have a proven efficacy in local treatment of CL with the view of developing this method to a method for screening of intralesionally administered drugs. Another development would be using intracellular amastigotes inside macrophages by the same method which may be more analogous to the real clinical situation.

The therapeutic animal study clearly demonstrated the effectiveness of orally administered zinc sulphate against lesions induced by inoculation of Leishmania parasite. The $\mathrm{ED}_{50}$ of zinc sulphate in this model was $59 \mathrm{mg} / \mathrm{kg}$.

The model employed has been used extensively in testing the effectiveness of drugs against strains of CL. Using this model Neal (1987) reported that the $\mathrm{ED}_{50}$ for sodium stibogluconate was $400 \mathrm{mg}$ of $\mathrm{Sb}^{\mathrm{v}} / \mathrm{kg}$ which gives a total dose of $4 \mathrm{~g} / \mathrm{kg}$ of the base. The $\mathrm{ED}_{50}$ for meglumin antimoniate in the same model was reported by the same author as $3.2 \mathrm{mg}$ of $\mathrm{Sb}^{\mathrm{v}} / \mathrm{kg}$ which gives an $\mathrm{ED}_{50}$ of 37.64 $\mathrm{mg} / \mathrm{kg}$ for the base. Other compounds tested were paromomycin which prevented the development of L. major induced lesions in mice at a high dose level (100 mg base $/ \mathrm{kg})$ while lower dose showed less effect.

The prophylactic study also clearly demonstrates that zinc sulphate administered prophylactically prevented the development of lesions of CL. The effectiveness of zinc against both promastigotes and amastigotes of both species of cutaneous leishmania as well as the immunomodulatory effects of zinc may account for the prophylactic role of zinc against CL. To verify the prophylactic effect of zinc clinically may be difficult, but prophylaxis against CL has not been tried before till now. Therefore this may be a new and interesting addition to the armamentarium of anti leishmanial drugs.

It may be argued that Old World CL is a relatively benign condition. But in opening this new perspective and with the possible extension of this work to visceral form i.e. Kala Azar as well as to the New World CL might be a step forward in the control of these forms. Initial results indicate that the visceral form of the parasite may also be sensitive in vitro (Najim et al. 1998).

In conclusion, this study provides evidence for the effectiveness of zinc sulphate against both strains of Leishmania causing Old World CL and encourage further study of the clinical effects of zinc sulphate both injected intralesionally and orally in treatment of CL.

\section{REFERENCES}

Adler S, Theodor O 1926. The identity of Leishmania tropica, Wright 1903 and Herpetomanas papatasi, Adler 1925. Ann Trop Med Parasit 20: 355-364.

Al-Ani ABJ 1992. Ultrastructure, Serial Cultivation and Chemotherapy of Visceral Leishmania Axenic Amastigotes and its Comparison with Promastigotes and Intracellular Amastigotes, $\mathrm{PhD}$ Thesis, College of Science, University of Baghdad, p. 105-120. 
Al-Bashir NT 1992. Comparative study of some enzymes of carbohydrate metabolism in Leishmania donovani and Leishmania major promastigotes, axenic amastigotes and in vivo amastigotes, p. 75. In Proceedings of the Sixth Congress, College of Medicine, University of Baghdad.

Al-Bashir NT, Rassam MB, Al-Rawi BM 1992. Axenic cultivation of amastigotes of Leishmania donovani and Leishmania major and their infectivity. Ann Trop Med Parasitol 86 : 487-502.

Al-Rubaiee AW 1990. Infra-red Therapy of Cutaneous Leishmaniasis (Baghdad Boil), Diploma Dissertation, College of Medicine, University of Baghdad, Iraq, p. 19-25.

Bassiouny A, El-Meshad M, Talaat M, Kutty K, Metawaa B 1982. Cryosugery in cutaneous leishmaniasis. $\mathrm{Br}$ J Dermat 107: 467-471.

Bryceson ADM 1987. Therapy in man, p. 848-903. InW Peters, R Killick- Kendrick (eds), The Leishmaniasis in Biology and Medicine, Academic Press, London.

Cousin RJ 1985 Absorption, transport and hepatic metabolism of copper and zinc: special reference to metallothionein and ceruloplasmine. Physiol Rev 65: 283-307.

Desjeux P 1992. Human leishmaniasis: epidemiology and public health aspects. World Health Statistic Quarterly 45: 267-275.

El-On J, Jacobs GP, Weinrauch L 1988. Topical chemotherapy of cutaneous leishmaniasis. Parasitol Today 4: 76-81.

Faris RM, Jarallah JS, Khoja TA, Al-Yaman MJ 1993. Intralesional treatment of cutaneous leishmaniasis with sodium stibogluconate antimony. Inter $J$ Dermatol 32 : 610-612.

Fridlender B, Chejanovsky N, Becker K 1978. Selective inhibition of herpes simplex virus type I DNA polymerase by zinc ion. Virology 81: 551-554.

Hearly MJP 1988. Estimation of $\mathrm{LD}_{50}$ without a computer. Parasitol Today 4: 25-27.

Jaffe CL, Grimaldi G, McMahon-Pratt D 1987. The cultivation and cloning of Leishmania, p. 47-90. In CM Morel, Genes and Antigens of Parasites: A Laboratory Manual, Fundação Oswaldo Cruz, Rio de Janeiro.

Kurban R, Al-Gindan Y, El-Hassem AM, Omer AHS 1986. Ketocanazole in cutaneous leishmaniasis: results of a pilot study. Saudi Med J 7: 596-604.

Najim RA, Sharquie KE, Turki KM, Farjo IB 1998. The in vitro effect of zinc sulfate against $L$. donovani. $J$ Fac Med (Baghdad) 40: 31-34.

Neal RA 1987. Experimental chemotherapy, p. 793-837. In W Peters, R Killick-Kendrik (eds), The Leishmaniasis in Biology and Medicine, Academic Press, London.

Neldner KH 1982a. Acrodermatitis enteropathica, p. 1012. In S Maddin, Current Dermatological Therapy, WB Saunders \& Co., Philadelphia.
Neldner KH 1982b. Zinc deficiency and the skin, p. 5054. In S Maddin, Current Dermatological Therapy, WB Saunders \& Co., Philadelphia.

Neouimine NI 1996. Leishmaniasis in the Eastern Mediterranean Region. East Mediterranean Health $\mathrm{J} 2$ : 94-101.

Pan AA 1984. Leishmania mexicana: serial cultivation of intracellular stage in a cell-free medium. Exp Parasitol 58: 72-80.

Pan AA, Pan SC 1986. Leishmania mexicana a comparative fine structure of amastigotes and promastigotes in vitro and in vivo. Exp Parasitol 62: 254-265.

Prasad A 1979 Zinc in Human Nutrition, CRC Press, Florida, p. 50-58.

Prasad A 1985. Zinc disorders, p. 759-765. In J Ston, Dermatology, Immunology and Allergy, CV Mosbey \& Co., St Louis.

Rahim GF, Tatar IH 1966. Oriental sore in Iraq. Bull of End Dis (Baghdad) 8: 29-54.

Sharquie KE 1993. A new intralesional therapy of cutaneous leishmaniasis with hypertonic sodium chloride solution, Iraqi Central Organization for Specification \& Quality Control/Patent Section, Patent No. 2459, Baghdad, Iraq.

Sharquie KE 1995. A new intralesional therapy of cutaneous leishmaniasis with hypertonic sodium chloride solution. J Dermatol (Japan) 22: 732-737.

Sharquie KE, Al-Azzawi KE 1996 Intralesional therapy of cutaneous leishmaniasis with $2 \%$ zinc sulphate solution, Iraqi Central Organization for Specification \& Quality Control/Patent section, Patent number 2583, Baghdad, Iraq.

Sharquie KE, Al-Hamamy HR, El-Yassin DI 1998. Treatment of cutaneous leishmaniasis by direct current therapy. The Baghdadin device. J Dermatol (Japan): 25: 234-237.

Sharquie KE, Al-Talib K, Chu AC 1988. Intralesional therapy of cutaneous leishmaniasis with sodium stibogluconate antimony. Brit J Dermatol 119: 5357.

Sharquie KE, Najim RA, Farjou IB 1997. A comparative control trial of intralesionally administered zinc sulfate, hypertonic saline, chloride and pentavalent antimony compounds against acute cutaneous leishmaniasis. Clin Exp Dermatol 22: 169-173.

Trotter ER, Peters W, Robinson BL 1980. The experimental chemotherapy of leishmaniasis. VI The development of rodent models for cutaneous infections with L. major and L. mexicana amazonensis. Ann Trop Med Parasitol 74: 299-319.

Wirth JJ, Frakur PJ, Kierszenbaum F 1989. Zinc requirement for macrophage function: effect of zinc deficiency on uptake and killing of protozoan parasite. Immunology 68: 114-119.

WHO 1984. The Leishmaniasis: Report of a WHO expert committee. Tech. Rep. Series WHO No. 701, World Health Organization, Geneva, p. 105-107. 
838 Zinc Treatment for Cutaneous Leishmaniasis - Rafid A Najim et al. 